\title{
5 Studienverhaltens- und Ergebnisindikatoren struktureller Studierbarkeit
}

In den vorangehenden Kapiteln wurde gezeigt, dass die zeitlichen Restriktionen von Studierenden deren Studierverhalten beeinflussen. Dieses Kapitel verbindet nun diese Ergebnisse und die Beschreibungen der Studienstrukturen aus Kapitel 4 mit der Forschung zu Studierverhalten und Studienerfolg. Es werden konkrete Indikatoren zur Messung des Studierverhaltens und der Ergebnisse struktureller Studierbarkeit hergeleitet sowie Hypothesen $\mathrm{zu}$ dem Modell struktureller Studierbarkeit (siehe Abbildung 3) - also den Zusammenhängen zwischen zeitlichen Restriktionen, Studienstrukturen und Studierverhalten bzw. Studienerfolg - aufgestellt. Die angenommenen Zusammenhänge sind in Abbildung 6 zusammengefasst.

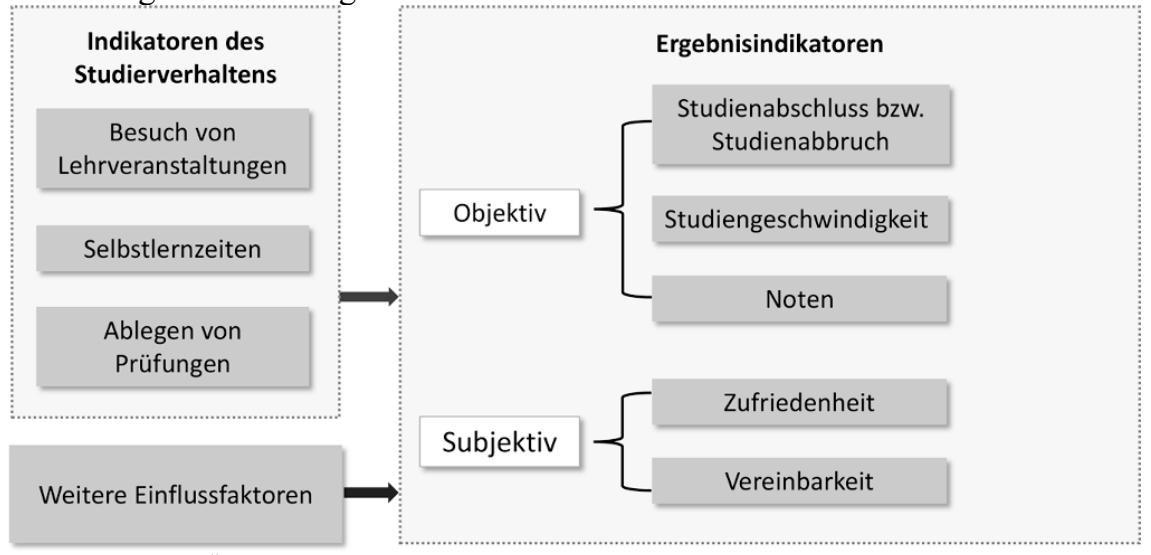

Abbildung 6 Überblick über den Inhalt von Kapitel 5.

Im folgenden Unterkapitel werden die Indikatoren für das Studierverhalten näher analysiert. Im Anschluss daran stehen im Unterkapitel 5.2 die Ergebnisindikatoren Studienabbruch, Studienzeit und Studienzufriedenheit im Mittelpunkt.

\subsection{Indikatoren des Studierverhaltens}

Es stellt sich zunächst die Frage, wie das Studierverhalten gemessen werden kann. Entsprechende Studierverhaltensindikatoren, welche das Lernen in Lehrveranstaltungen und zu Hause im Selbststudium vorsehen, können sich an der 
Einteilung der Arbeitsbelastung von Studierenden orientieren (KMK 2010, S. 2). $\mathrm{Ob}$ und in welchem Umfang Studierende Lehrveranstaltungen besuchen, ist daher der erste Indikator für das Studierverhalten. Widerholungs- und Anwendungsprozesse oder auch die eigenständige Erarbeitung von Inhalten finden in der Selbstlernzeit statt, weshalb diese als zweiter Indikator genutzt wird. Neben der zeitlichen Investition der Studierenden in das Studium kann der Studienfortschritt durch das Ablegen von Modulprüfungen sichtbar gemacht werden. Die Prüfungen sind gleichzeitig eine Messung des Kompetenzzuwachses bzw. Lernerfolgs. Dieser Aspekt ist daher ein dritter Indikator für das Studierverhalten.

\subsubsection{Besuch von Lehrveranstaltungen}

Im Kontext von Präsenzstudiengängen findet die Vermittlung kognitiver, methodischer und sozialer Kompetenzen primär in Lehrveranstaltungen statt. Die Studienstruktur beeinflusst den Besuch von Lehrveranstaltungen, weil die Lage und der Umfang von Präsenzzeiten im Zusammenspiel mit zeitlichen Restriktionen von Studierenden auf die Anwesenheit einwirken (van den Berg und Hofman 2005, S. 427). Die Teilnahme wird dann eingeschränkt, wenn Studierende aufgrund ihrer außerhochschulischen Verpflichtungen nicht teilnehmen können oder sich die Veranstaltungen überschneiden. Die Studienstruktur sowie zeitliche Restriktionen können auch die subjektive Studienbelastung der Studierenden beeinflussen und möglicherweise die Probleme beim Besuch von Lehrveranstaltungen moderieren (Groß und Boger 2011, S. 162).

Doch warum ist der Besuch von Lehrveranstaltungen ein wichtiger Indikator,

um das Studierverhalten zu messen? Seltene Anwesenheit bei Lehrveranstaltungen kann - wenn die Kompetenzen nur schwer anders erworben werden können oder die Motivation der Studierenden leidet - den Lernfortschritt reduzieren. Mehrere Studien finden einen schwachen positiven Effekt von regelmäßiger Teilnahme auf die erreichten Noten (Arulampalam et al. 2012, S. 370; Cohn und Johnson 2006, 225 ff.; Devadoss und Foltz 1996, 504 ff.; Dobkin et al. 2010, 570 ff.). Diese Ergebnisse könnten den Effekt der Veranstaltungsteilnahme jedoch überschätzen. Denn die Leistungsfähigkeit von Studierenden hängt mit der Anwesenheit zusammen (Dobkin et al. 2010, S. 572), und motivierte und interessierte Studierende nehmen häufiger an Präsenzveranstaltungen teil (Andrietti und Velasco 2015, S. 250; Devadoss und Foltz 1996, S. 504). Zahlreiche weitere Faktoren beeinflussen die Veranstaltungsteilnahme, so die zeitliche Lage der Veranstaltung und die Wahrnehmung der Kompetenz der Lehrenden (Arulampalam et al. 2012, S. 369). 
Auch wenn der Zusammenhang zwischen Veranstaltungsteilnahme und Noten gering ist, ist die Möglichkeit der Veranstaltungsteilnahme von großer Wichtigkeit für Studierende. Denn je nach Studienfach und zu erwerbenden Kompetenzen ist die Lehrveranstaltung ein wichtiger Lernort. Wie wichtig die Lehrveranstaltung für den Kompetenzerwerb ist, hängt von zwei Faktoren ab. Erstens spielt die Anwesenheit in Fächern, in denen entweder das Wissen unzulänglich in Büchern, Artikeln oder anderen Informationsquellen aufbereitet ist oder in denen zur Kompetenzentwicklung der Diskurs in der Gruppe oder die Anwendung oder das Erleben nur in Präsenz möglich ist (z. B. Labore, Exkursionen), eine wichtige Rolle für den Lernfortschritt. Möglicherweise zeigen die Studien von Dobkin et al. (2010) und Andrietti und Velasco (2015) nur deshalb einen geringen Zusammenhang zwischen Anwesenheit und Noten, weil sie in Statistik- bzw. BWL-Veranstaltungen durchgeführt wurden, in denen sehr gute Fach- und Lehrbücher für das Selbststudium existieren. Zweitens ist für die Studierenden je nach Lernstrategie (Wild und Wild 2012, S. 12 ff.) die Lehrveranstaltung unterschiedlich wichtig für den Kompetenzerwerb. Lehrveranstaltungen haben schließlich im Kontext der Motivation auch das Ziel, die Studierenden zu regelmäßigem Lernen anzuhalten und die Reflexion der Studierenden über die Relevanz des Lerngegenstandes zu fördern. Wie stark die Studierenden auf eine entsprechende Unterstützung durch Lehrende angewiesen sind, hängt auch von ihren Vorerfahrungen ab. Denn ältere und berufstätige Studierende erkennen den Nutzen des Lerngegenstandes stärker selbst und können sich besser motivieren, da sie sich oft zielgerichteter für ein Studium entscheiden (Donaldson und Graham 1999, S. 28 ff.). Diese Fähigkeit kommt den berufstätigen Studierenden insbesondere in solchen Fächern zugute, die sich gut für eigenständiges Erarbeiten der Lerninhalte eignen, da sie in solchen Fächern verpassten Lernstoff besser nachholen können.

Zusammenfassend lässt sich sagen, dass die Anwesenheit bei Lehrveranstaltungen für das Lernen oft von großer Relevanz ist. Wie wichtig die Lehrveranstaltungen für die Studierenden sind, hängt allerdings von ihren Lernstrategien, ihren Vorerfahrungen und dem Lerngegenstand selbst ab. Daher ist eine Abwesenheit nicht generell nachteilig. Vielmehr haben diejenigen Studierenden Nachteile, die aufgrund von unpassenden Studienstrukturen oder zeitlichen Restriktionen unfreiwillig nicht an Veranstaltungen teilnehmen.

\subsubsection{Aufbringen von Selbstlernzeit}

Es gibt drei Arten des Selbststudiums: begleitetes, individuelles und freies Selbststudium. Das begleitete Selbststudium orientiert sich an Aufgaben, die für ein Modul zu erfüllen sind, und kann entweder alleine oder in Begleitung durch 
Lehrende und andere Studierende geschehen. Beim individuellen Selbststudium üben Studierende, bereiten Veranstaltungen vor und nach und lernen für Prüfungen. Das freie Selbststudium ist unabhängig von der konkreten Lehrveranstaltung (Landwehr und Müller 2008, S. 21).

Die Selbstlernzeit ist für den Studienerfolg relevant, da eine längere Selbstlernzeit positiv mit besseren Noten zusammenhängt (Andrietti und Velasco 2015, S. 252). Die ZeitLAST-Studien zeigen, dass der größte Teil der Selbstlernzeit fokussiert auf die Prüfungsvorbereitung genutzt wird (Metzger 2011, S. 241). Die Intensität und der Umfang des Selbststudiums hängen, der Theorie der Selbstbestimmung nach Deci und Ryan entsprechend, von einer hohen intrinsischen Motivation und einer guten Selbstorganisation ab (Ryan und Deci 2000, S. 68). Die Literatur zur Vereinbarkeit von Studium, Familie und Beruf zeigt darüber hinaus, dass hohe außerhochschulische Belastungen die Selbstlernzeit reduzieren (Beerkens et al. 2011, S. 680; Dippelhofer-Stiem 2012, S. 420; Oosterbeek und van den Broek 2009, S. 176).

Neben zeitlichen Restriktionen und weiteren individuellen Faktoren beeinflusst auch die Studienstruktur die Selbstlernzeit. Schulmeister und Metzger (2011, S. 119) zeigen, dass eine Vielzahl von Einzelveranstaltungen und eine damit einhergehende hohe Anzahl von SWS und Prüfungen dazu führen, dass den Studierenden pro Veranstaltung bzw. Thema weniger potenzielle Selbstlernzeit zur Verfügung steht. Andererseits ist der Stundenplan durch die zweistündigen Lehrveranstaltungen mit vielen kurzen Zeitlücken zerrissen. Studierende müssen sich mehrfach am Tag auf neue Themen einstellen und haben wenige Zeitfenster, in denen sie längere Zeit ein Thema im Selbststudium bearbeiten können (Schulmeister und Metzger 2011, S. 103 ff.) Längere freie Zeiten und eine geringere Anzahl an SWS und Prüfungen können diesen strukturellen Defiziten begegnen und mehr Freiräume für das Selbststudium schaffen.

Zusammenfassend lässt sich festhalten, dass unter anderem zeitliche Restriktionen den Umfang des Selbststudiums beeinflussen. Je nach Studienstruktur können die Studierenden ihre zeitlichen Ressourcen für das Selbststudium einsetzen. Da die optimale Dauer des Selbststudiums ist sehr individuell ist und stark von den Vorkenntnissen und der Motivation der Studierenden abhängt, wird im Modell struktureller Studierbarkeit nicht der Umfang in Stunden gemessen. Vielmehr stehen die individuell bewerteten Probleme beim Aufbringen der Selbststudienzeit im Mittelpunkt der Analysen.

\subsubsection{Ablegen von Prüfungen}

Die Überprüfung der Lernzielerreichung wird durch studienbegleitende Modulprüfungen durchgeführt. Prüfungen sind daher dazu geeignet, den 
Lernfortschritt und die Studienschwierigkeiten von Studierenden zu beobachten. Infolge einer geringen Selbstlernzeit oder Anwesenheit bei Veranstaltungen kann sich das Ablegen von Prüfungen reduzieren. Bisher liegen allerdings keine Studien dazu vor, welche Faktoren den Antritt einer Prüfung beeinflussen. Da das Ablegen von Prüfungen stark mit der Studiengeschwindigkeit zusammenhängt, kann dieser Indikator hilfsweise für die Hypothesenentwicklung genutzt werden. Die Dauer des Studiums hängt dabei unter anderem mit zeitlichen Restriktionen durch Erwerbstätigkeit und Sorgeaufgaben und der Studienstruktur zusammen. Doch auch zahlreiche andere Faktoren, wie die Motivation, zusätzliches Engagement oder Praktika und die Studienfinanzierung, beeinflussen die Studiendauer (zu entsprechender Literatur siehe Kapitel 5.2.2.). Ob Studierende Prüfungen so ablegen wie im Studienverlaufsplan vorgesehen, hängt also von vielen Faktoren ab. Das Ablegen einer geringeren Anzahl von Prüfungen als von der Hochschule vorgesehen kann von den Studierenden so vorgesehen sein. Als Indikator für das Studierverhalten kommt daher die Einschätzung zur Anwendung, ob Studierende subjektiv Probleme beim Ablegen von Prüfungen zum geplanten Zeitpunkt haben.

\subsubsection{Hypothesen zum Studierverhalten}

Zusammenfassend und unter Berücksichtigung der Forschungsergebnisse aus Kapitel 1 lässt sich sagen, dass Studierende mit zeitlichen Restriktionen Schwierigkeiten damit haben, trotz ihrer Verpflichtungen regelmäßig bei Lehrveranstaltungen anwesend zu sein und genügend Selbstlernzeit aufzubringen. $\mathrm{Ab}$ welchem Umfang der zeitlichen Restriktion diese Vereinbarkeitsprobleme virulent werden, ist nicht eindeutig belegt und zu prüfen.

\section{H2: Zeitliche Restriktionen hängen negativ mit der Teilnahme an Lehrveranstaltungen, dem Aufbringen von Selbstlernzeit und der Prüfungsteilnahme zusammen.}

Es gibt Hinweise darauf, dass Studienstrukturen, die zu den Bedarfen der Studierenden mit zeitlichen Restriktionen passen, die Vereinbarkeit von Studium und Verpflichtungen außerhalb des Studiums sowie das Studierverhalten verbessern können. Da die zeitlichen Restriktionen sehr heterogen sind, sollten die Studienstrukturen flexibel und damit für unterschiedliche Bedarfe ausgestaltet sein (siehe Kapitel 4). Bisher wurde der Zusammenhang zwischen Studienstrukturen und Studierverhalten als direkter Effekt untersucht. Das Modell struktureller Studierbarkeit nimmt hingegen einen Interaktionseffekt der Studienstruktur an. Es ist zu erwarten, dass in flexiblen Studienstrukturen die 
negativen Auswirkungen von zeitlichen Restriktionen geringer sind als in unflexiblen Strukturen.

H3: Studienstrukturen mit hoher Flexibilität reduzieren den

Zusammenhang zwischen zeitlichen Restriktionen und Problemen beim

Besuch von Lehrveranstaltungen und Prüfungen sowie dem Aufbringen von Selbstlernzeit.

\subsection{Ergebnisindikatoren}

Die Indikatoren für die Ergebnisse struktureller Studierbarkeit werden im Folgenden aus Studienerfolgskriterien hergeleitet. Die Europäische Kommission analysiert Studienerfolg und Studienabbruch sowie Unterschiede zwischen den Ländern und definiert Studienerfolg wie folgt:

"Study success comprises all major achievements of students in the higher education system, including dropout/retention, completion of a degree and time-to-degree" (European Commission 2015, S. 24).

Der Studienabschluss und eine geringe Anzahl an Studienabbrecherinnen und Abbrechern sind auch in Deutschland die am häufigsten genannten Studienerfolgskriterien (Berthold et al. 2015, S. 16; Statistisches Bundesamt 2014). Die Studienerfolgsfaktoren lassen sich in zwei Kategorien einteilen. Einerseits werden aus statistischen Studierendendaten objektive Indikatoren wie die Studienzeit, die Abschlussnote oder die Abbruchquote gewonnen. Dies sind auch die von der Europäischen Kommission genutzten Kennzahlen (siehe das obige Zitat). Andererseits werden Studierende subjektiv nach ihrer Zufriedenheit oder der Vereinbarkeit zwischen Studium und anderen Verpflichtungen gefragt (Berthold et al. 2015, S. 16). In diesem Kapitel werden nur diejenigen Kriterien näher analysiert und als Ergebnisindikatoren genutzt, welche empirisch belegt durch die Studienstruktur beeinflusst werden. Die Auswahl wird im Folgenden begründet.

Die Studienbedingungen inklusive der Studienstruktur wirken neben individuellen Faktoren wie beispielsweise Berufstätigkeit und Sorgeaufgaben auf den Studienabbruch ein (Heublein et al. 2010, S. 14). Daher ist der Studienabbruch ein Ergebnisindikator für strukturelle Studierbarkeit. Darüber hinaus weisen die Literatur und die Hochschulpolitik die Studiendauer (Jirijahn 2007, S. 287) oder den Studienfortschritt (Sedlacek 2004, S. 204; van den Berg und Hofman 2005, S. 427) als Erfolgskriterium aus. Der Einfluss von Studienstrukturen auf die Studiendauer wird von van den Berg und Hofman (2005) nachgewiesen. Auch zeitliche Restriktionen wie Erwerbstätigkeit verlängern insgesamt die 
Studiendauer (Brandstätter und Farthofer 2003, S. 140; Jirijahn 2007, S. 306; Sprietsma 2015, S. 12). Aus diesem Gründen geht die Studienzeit als Ergebnisindikator in die weiteren Analysen ein. Die Studienzeit und der Studienabbruch sind objektive Erfolgskriterien. Als subjektiver Ergebnisindikator struktureller Studierbarkeit eignet sich die Studienzufriedenheit, welche eng mit den Studienbedingungen und der Lebenssituation von Studierenden verknüpft ist (Blüthmann 2012, S. 292).

Zwei weitere Erfolgsfaktoren kommen nicht in Frage, da sie keinen eindeutigen Zusammenhang mit der strukturellen Studierbarkeit aufweisen. Neben dem Erwerb eines Hochschulabschlusses definieren einige Autoren auch die Noten des Studiums als Erfolgskriterium (Jirijahn 2007, S. 287). Da die Abschlussnoten jedoch statt auf Studienstrukturen primär auf individuelle Faktoren wie etwa die Abiturnote oder auf Fächerunterschiede zurückzuführen sind, geht dieser Indikator nicht in die Analysen dieser Arbeit ein (Müller-Bendict und Tsarouha 2011, S. 401; Sprietsma 2015, S. 11; Wissenschaftsrat 2012, S. 45 ff.). Die Vereinbarkeit von Studium, Erwerbstätigkeit und Familie liegt als Ergebnisindikator struktureller Studierbarkeit nahe, da sie das Zusammenpassen des Studiums mit den Anforderungen von Studierenden mit zeitlichen Restriktionen fokussiert. Da relevante Aspekte der Vereinbarkeitseinschätzung jedoch schon in den Studierverhaltensindikatoren (Schwierigkeiten beim Besuch von Lehrveranstaltungen, Aufbringen von Selbstlernzeit) enthalten sind, würden ähnliche Variablen doppelt in die Analysen eingehen. Dies würde bei der empirischen Analyse Modellverstöße erzeugen, sodass die Vereinbarkeit nicht als Ergebnisindikator genutzt werden kann.

Was die Literatur zu den Ergebnisindikatoren betrifft, ist zu beachten, dass die bisherige Forschung überwiegend den direkten Zusammenhang zwischen Einflussfaktoren wie Erwerbstätigkeit, Elternschaft, Studienbedingungen oder Motivation auf den Studienerfolg fokussiert. Das Konzept der strukturellen Studierbarkeit geht hingegen davon aus, dass der Studienerfolg über das Studierverhalten moderiert wird. Dennoch wird auch Literatur zitiert, in der ein direkter Zusammenhang angenommen wird.

\subsubsection{Studienabbruch}

Der Studienabbruch ist im Vergleich mit den anderen Ergebnisindikatoren am besten erforscht. Dabei gibt es differierende Definitionen eines Studienabbruchs. In den USA wird darunter das Verlassen einer Institution verstanden (SchröderGronostay und Daniel 1999, S. 213), in Deutschland hingegen das Verlassen des gesamten Hochschulsystems ohne Hochschulabschluss (Hörner 1999, S. 6). Der Schwund hingegen bezeichnet analog der US-amerikanischen Definition das 
Verlassen der Hochschule ohne Abschluss und bezieht sich auf Hochschul- und Fachwechsler sowie Studienunterbrecher (Lewin 1999, S. 17).

Theorien für den Studienabbruch suchen unterschiedliche Zugänge und können in ,soziologische, organisationstheoretische, psychologische oder ökonomische Erklärungsmodelle" untergliedert werden (Schröder-Gronostay 1999, S. 218). Die ökonomischen Erklärungsperspektiven beschäftigen sich insbesondere mit der Studienfinanzierung, die psychologischen Perspektiven mit der Selbstwahrnehmung der akademischen Fähigkeiten, Arbeitshaltungen sowie der Wertigkeit des Hochschulabschlusses für die Studierenden (z. B. Gold 1988, S. 63).

Die soziologischen Abbruchtheorien sind Grundlage zahlreicher Abbruchforschungen aus den USA und stammen unter anderem von Tinto (1993) und Spady (1970). Tinto entwickelte eine der am häufigsten zitierten Abbruchtheorien. Sie besagt, dass eine hohe soziale und akademische Integration zu einer starken Identifikation mit der Hochschule führt. Diese drückt sich darin aus, dass die oder der Studierende das Studium mit einem Abschluss beendet und sich loyal gegenüber der Hochschule verhält (Tinto 1975, S. 95). Tinto berücksichtigt auch individuelle Aspekte wie den finanziellen und sozialen Hintergrund sowie Bildungserfahrungen. Darüber hinaus geht er davon aus, dass es sich bei der Entscheidung für oder gegen einen Studienabbruch um eine Abwägung zwischen mehreren Alternativen (Verbleib, andere Tätigkeit, anderes College) handele. Dabei berücksichtigen die Individuen laut Tinto bei der Entscheidung für oder gegen ein Verlassen der Institution auch die Zeit, die Energie und die Ressourcen, die sie in das Studium investieren.

Für deutsche Hochschulen sind die Unterschiede zum US-amerikanischen Hochschulsystem zu bedenken. Die Loyalität zur Hochschule wird in den USA stark durch Traditionen und das Image einer (Elite-)Universität sowie durch das Leben auf dem Campus beeinflusst. Die Studiensituation in Deutschland zeichnet sich hingegen durch das Wohnen außerhalb des Campus und die geringere Rolle von außercurricularen Aktivitäten aus (Beekhoven et al. 2002, S. 595; Gilardi und Guglielmetti 2011, S. 36). Darüber hinaus geht Tinto von traditionellen Vollzeitstudierenden aus, deren soziale Integration sich beispielsweise in der intensiven Teilnahme an (studentischen) Organisationen und Aktivitäten ausdrückt. Dies können Studierende mit starken zeitlichen Restriktionen nicht leisten. Mehrere Artikel untersuchen daher, ob für diese Studierende andere Erklärungsmuster für den Studienabbruch heranzuziehen sind.

Bean und Metzner (1985) haben in diesem Kontext ein Studienabbruchmodell für nicht-traditionelle Studierende entworfen. Das Modell geht von geringeren Präsenzzeiten auf dem Campus aus, da ältere und berufstätige Studierende außerhalb der Hochschule leben. Daher spielt die soziale Integration 
bei Bean und Metzner eine geringere Rolle; die Haupteffekte sehen die Autoren in den Lebensumständen (z. B. Alter, Gender, Lebenssituation und finanzieller Hintergrund), der akademischen Leistung und deren Wahrnehmung durch die Studierenden. Das Studierverhalten und die Anwesenheit bei Lehrveranstaltungen beeinflusst Noten und Zufriedenheit sowie indirekt den Studienabbruch. Dem Akt des Studienabbruchs geht in diesem Modell der Wunsch voraus, die Hochschule zu verlassen. Die Autoren prüfen ihr Modell empirisch und weisen die Note im Studium, den Abbruchwunsch, den Teilzeitstatus, die Anwesenheit bei Lehrveranstaltungen und die Leistung in der Highschool als direkte Haupteffekte aus (Metzner und Bean 1987, S. 31). Wie von den Autoren antizipiert, spielt die soziale Integration keine signifikante Rolle für den Studienabbruch. Die Übertragbarkeit auf die Situation in Deutschland ist jedoch aufgrund der Unterschiede im Hochschulsystem und der Erhebung an nur einer einzigen Universität mit Vorsicht zu interpretieren. So zeigen neuere Ergebnisse aus Italien, dass eine schlechte soziale Integration insbesondere für berufstätige Studierende das Studienabbruchrisiko erhöht (Gilardi und Guglielmetti 2011, S. 44).

Für den deutschen Kontext ist Studienabbruchkonzept von Heublein et al. (2010) relevant, welches zeitliche Restriktionen und den Abbruchzeitpunkt berücksichtigt (siehe Abbildung 7). Die Beschriftungen in der Abbildung wurden zur besseren Lesbarkeit leicht gekürzt und der Aspekt des Abbruchzeitpunktes neu in die Grafik aufgenommen. Das Konzept passt die aus den Forschungen in den USA bekannten Aspekte dem deutschen Hochschulsystem an. Es geht davon aus, dass auch die Situation vor dem Studium eine Rolle beim Studienabbruch spielt, so die Erwartungen an das Studium und die Studienvoraussetzungen. Während des Studiums beeinflussen die Leistungsfähigkeit und Motivation, die Integration sowie die Studienbedingungen und Studienstrukturen die Bewertung des Studiums. In der konkreten Entscheidungssituation wägen die Studierenden die finanziellen Ressourcen, die Übereinstimmung mit den Lebensbedingungen und die Alternativen zum Studium ab. Beratung durch die Hochschule oder Personen und Institutionen außerhalb der Hochschule kann den Entscheidungsprozess begleiten. 


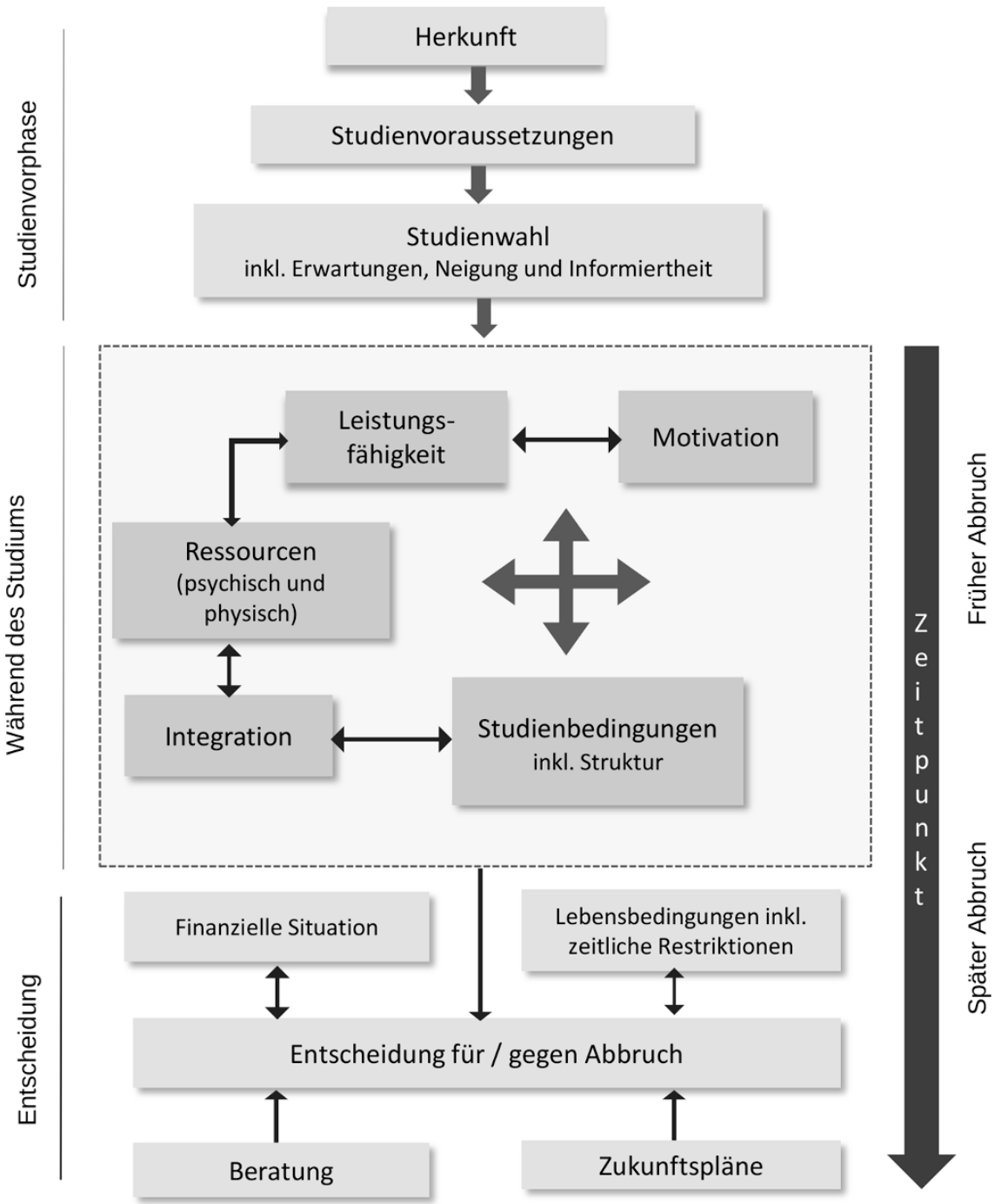

Abbildung 7 Studienabbruch: Gründe, Entscheidungsprozess und Abbruchzeitpunkt. Adaptiert nach Heublein et al. 2010, S. 14 und S. 50 ff.

Doch warum ist der Studienabbruch als Ergebnisindikator für die strukturelle Studierbarkeit interessant? Mehrere Forschungsergebnisse weisen auf die Rolle der Studienbedingungen bei der Entscheidung für oder gegen das Fortführen des 
Studiums hin. Die Studienstruktur ist dabei in der Regel ein Aspekt der Studienbedingungen. Pohlenz und Tinsner (2004, S. 110) zeigen, dass schlechte Studienbedingungen von den Studierenden als Hauptabbruchgrund genannt werden. Die Studienbedingungen sind hier die Abstimmung des Lehrangebots untereinander, Rahmenbedingungen wie Öffnungszeiten der Bibliothek und die Qualität von Lehrveranstaltungen. Georg weist als wichtige Studienbedingung die Lehrqualität aus, welche den Studienabbruch beeinflusst (Georg 2008, S. 200). Beide Forschungsarbeiten sehen jedoch den Einfluss der Studienbedingungen auf den Studienabbruch als eher gering an. Demgegenüber weisen andere Arbeiten den Studienbedingungen ein größeres Gewicht zu. Blüthmann et al. sehen die Studienbedingungen und die darin enthaltene Studienstruktur (u. a. Wahlmöglichkeiten, Verschulung des Studiums) als zweitgrößten Einflussfaktor auf die Abbruchneigung (Blüthmann et al. 2008, S. 423; Blüthmann et al. 2011, S. 113). Auch Ergebnisse der bundesweiten Exmatrikuliertenbefragung des DZHW (ehemals HIS) unterstützen dies. Heublein et al. (2010, S. 19) zeigen, dass die Studienbedingungen bei fast allen Studienabbrüchen eine Rolle spielen (Abbildung 8). Ausschlaggebend sind die schlechten Studienbedingungen aber für 12 Prozent der Abbrechenden. Neben den unterschiedlichen Datengrundlagen und Auswertungsmethoden kann die Unterscheidung in allgemeine und ausschlaggebende Gründe einen Ansatzpunkt für die tatsächliche Bedeutung der Studienstrukturen oder Studienbedingungen bieten. So lösen schlechte Studienbedingungen überwiegend im Zusammenspiel mit anderen Faktoren Studienabbrüche aus. Interessant ist, dass die Studienbedingungen im Jahr 2008 um vier Prozentpunkte häufiger als ausschlaggebender Abbruchgrund genannt wurden als acht Jahre zuvor (Heublein et al. 2010, S. 19 f.). Dieser Befund könnte darauf hindeuten, dass in den gestuften und vergleichsweise stark strukturierten Bachelor- und Masterstudiengängen die Studienbedingungen kritischer oder unpassender eingeschätzt werden. 
Leistungsprobleme

Finanzielle Probleme

Mangelnde Studienmotivation

Studienbedingungen

Prüfungsversagen

Berufliche Neuorientierung

Familiäre Probleme

Krankheit

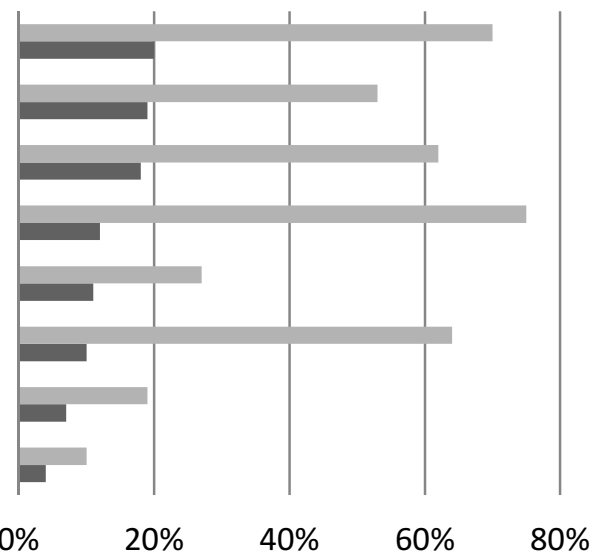

Allgemeine Begründung

Ausschlaggebende Gründe

Abbildung $8 \quad$ Allgemeine und ausschlaggebende Studienabbruchgründe für den Jahrgang 2008. Allgemeine Gründe: Mehrfachantwort möglich. Eigene Darstellung mit Daten aus Heublein et al., 2010, S. $19 \mathrm{f}$.

Neben diesen institutionellen und studienstrukturellen Faktoren beeinflussen vor allem individuelle Aspekte den Studienabbruch. Betrachtet man zunächst die Studierenden mit zeitlichen Restriktionen, so sind finanzielle oder familiäre Gründe und die Vereinbarkeit von Studium und außerhochschulischen Verpflichtungen bei diesen Gruppen ein häufiger Abbruchgrund (Brandstätter und Farthofer 2003, S. 141; Heublein et al. 2010, S. 24 ff., 41 ff.; Lewin 1999, S. 21). Die zeitlichen Restriktionen können in Interaktion mit der Studienstruktur daher den Studienabbruch verstärken. Gelingt die Vereinbarkeit, sind Studierende nach Meulemann (1991, S. 232), ,gut vor Studienabbruch geschützt“.

Einig sind sich die Autoren, dass eine hohe Studienmotivation die Abbruchwahrscheinlichkeit reduziert. Blüthmann et al. (2011, S. 113) sehen im fehlenden Fachinteresse den Hauptgrund für eine selbsteingeschätzte Studienabbruchneigung. Pohlenz und Tinsner (2004, S. 78) analysieren Exmatrikulierte der Universität Potsdam. Die Autoren weisen unerfüllte Erwartungen in Bezug auf das Fach und die Studienbedingungen als einen von vier Faktorenbündeln für den Studienabbruch aus. Die Studienvoraussetzungen in Form von Noten und die fachlichen Schwierigkeiten bzw. die Schwierigkeiten beim Lernen beeinflussen das Abbruchrisiko ebenfalls (Blüthmann et al. 2011, 113 f.; Heublein et al. 2010, S. 19; Pohlenz und Tinsner 2004, S. 79; Sedlacek 
2004, S. 205). Eine gute Integration in die Hochschule reduziert das Risiko eines Studienabbruchs (Gilardi und Guglielmetti 2011, S. 42; Heublein et al. 2010, S. 117; Tinto 1975, S. 103). Nach Meeuwisse et al. (2010, S. 536) beinhaltet die Integration jeweils eine formale Interaktion in Lernsituationen (Lerngruppen, Lernen und Beratung durch Lehrende) sowie eine informelle Interaktion (persönliche Kontakte, Freundschaften) mit Kommilitonen (sozial) und Lehrenden (akademisch) (siehe auch Severiens et al. 2006).

Die Studienabbruchgründe können ebenfalls anhand des Zeitpunktes des Studienabbruchs untersucht werden. Heublein et al. (2010, S. 47) zeigen zunächst, dass sich der durchschnittliche Zeitpunkt von 7,6 Semester im Jahr 2000 auf 6,3 Semester in 2008 reduziert hat. Dieser Effekt sei allein auf die Einführung von Bachelorstudiengängen zurückzuführen, bei denen der Durchschnitt des Abbruchzeitpunktes bei 2,9 Semestern liegt. Nach Schindler (1999, S. 164) kommt es zu einem „frühen“ Abbruch in den ersten beiden Fachsemestern. Die Abgrenzung zwischen „frühen“ und „späten“ Abbrechern ist jedoch schwierig. Tendenziell geben frühe Abbrecher als Gründe schlechte Studienbedingungen, der Wunsch nach einer Neuorientierung, Leistungsprobleme und fehlende Motivation an (Gold 1988, S. 118; Heublein et al. 2010, S. 52). Frühe Abbrecher sind zwar fleißig und leistungswillig, aber auch emotional unsicherer und weniger durchsetzungsfähig als Studierende, die an der Hochschule verbleiben. Schindler zeigt am Beispiel der Universität Regensburg, dass die frühen Abbrecher eine weniger bewusste und informierte Studienwahl getroffen haben und etwas schlechter sozial integriert sind (Schindler 1999, S. 165) Der seltenere und auch qualitativ schlechtere Kontakt zu Kommilitonen erschwert die Lösung von fachlichen und organisatorischen Studienschwierigkeiten, die häufig im Austausch mit anderen Studierenden gefunden werden. Späte Abbrecher geben als Gründe eher finanzielle oder familiäre Gründe sowie Prüfungsversagen an (Heublein et al. 2010, S. 52). Sie lassen sich verallgemeinernd als psychisch stabil (Gold 1988, S. 117) und gleichzeitig weniger leistungsfähig beschreiben (Schindler 1999, S. 165). Späte Abbrecher können Leistungsschwierigkeiten aushalten; wenn der Prüfungserfolg sich nicht einstellt, kommt es jedoch zur freiwilligen oder erzwungenen Exmatrikulation.

Methodisch existieren einige Herausforderungen bei der Erfassung von Studienabbrüchen. Um das Ereignis des Studienabbruchs selbst beobachten zu können, werden Längsschnittstudien während des Studiums und nach dem Studium durchgeführt. Eine Alternative hierzu bilden retrospektive Befragungen oder die Analyse der Studienabbruchneigung. Der zweite Ansatz kann insbesondere durch Querschnittsbefragungen aktuell Studierender erfasst werden. Dabei ist zu beachten, dass eine entsprechende Abbruchneigung nicht unmittelbar $\mathrm{zu}$ einem Studienabbruch führt. Gold zeigt, dass lediglich etwa 20 Prozent der 
abbruchgefährdeten Personen ihr Studium tatsächlich abbrechen. Von den Abbrechern hingegen hat der überwiegende Teil von 80 Prozent vorher eine Studienabbruchneigung geäußert (Gold 1988, S. 122). Forschung zu nichttraditionellen Studierenden weisen die Abbruchneigung als zweitgrößten Effekt auf den Abbruch aus (Metzner und Bean 1987, S. 31). Die unterschiedlichen Messungen führen zu Schwierigkeiten in der Vergleichbarkeit der zuvor darstellen Ergebnisse.

Zusammenfassend lässt sich feststellen, dass die Studienbedingungen - und als Teilbereich hiervon die Studienstrukturen - einen der Einflussfaktoren auf den Studienabbruch oder die Studienabbruchneigung darstellen. Auch die zeitlichen Restriktionen (z. B. Berufstätigkeit) erhöhen die Wahrscheinlichkeit eines Studienabbruchs. Das Modell struktureller Studierbarkeit geht jedoch nicht von einem direkten Zusammenhang zwischen Studienstrukturen und zeitlichen Restriktionen auf den Studienabbruch aus. Vielmehr ist dieser durch das Studierverhalten moderiert. Georg (2008, S. 200) unterstützt diese Annahme, indem er den Einfluss eines von Regelmäßigkeit und Lernaktivität geprägten Studierverhaltens (z. B. Besuch von Lehrveranstaltungen, Selbstlernzeit, Prüfungen) auf den Studienabbruch nachweist. Entsprechend den vorangegangenen Überlegungen wird folgende Hypothese in dieser Arbeit geprüft:

H4: Je mehr Schwierigkeiten Studierende beim Besuch von

Lehrveranstaltungen und Prüfungen sowie dem Aufbringen von

Selbstlernzeit haben, umso höher ist ihre Studienabbruchneigung.

\subsubsection{Studiengeschwindigkeit}

Die Studiengeschwindigkeit beschreibt die Dauer des Studiums in Relation zur vorgesehenen Regelstudienzeit. Diese wiederum ist durch gesetzliche Vorgaben weitestgehend festgelegt, da Bachelorstudiengänge aus sechs bis acht Semestern und Masterstudiengänge aus zwei bis vier Semestern bestehen sollen (KMK 2010, S. 2). Die Studiengeschwindigkeit wird im deutschsprachigen Raum seit vielen Jahren kritisch diskutiert, da die Regelstudienzeiten teilweise stark überschritten werden (Schawan 2010, S. 4). Besonders stark zeigen dies ältere Studien aus Österreich (Dell'amour und Landler 2002; Sedlacek 2004). Aber auch in Deutschland wird die Verringerung der Studiendauer als ein Element der BolognaReform politisch diskutiert (Wissenschaftsrat 2011, S. 9). Durch die Einführung des Workload-Konzepts versuchen die Hochschulen nun, die zu investierende Zeit für das Studium zu messen (Schulmeister und Metzger 2011, S. 14). Die Einflussfaktoren auf die Studiendauer sind dabei auf studentischer, institutioneller 
und rechtlicher sowie wirtschaftlicher Ebene anzusiedeln (van den Berg und Hofman 2005, S. 415). Die Studiengeschwindigkeit ist als Ergebnisindikator struktureller Studierbarkeit interessant, da sie im Rahmen der individuellen Faktoren von den zeitlichen Restriktionen und im Sinne der institutionellen Faktoren von den Studienstrukturen beeinflusst wird. Studien zu dem in dieser Arbeit angenommenen Zusammenhang zwischen dem Studierverhalten und der Studiendauer liegen allerdings kaum vor.

Zeitliche Restriktionen, etwa durch Berufstätigkeit oder Elternschaft, wirken als individueller Faktor auf die Studiendauer. Dabei hängt der Einfluss vom Umfang der Restriktionen ab. Einige Studien differenzieren nicht nach dem Umfang der Berufstätigkeit und stellen einen generellen Effekt von Berufstätigkeit auf die Studiendauer fest (Aina et al. 2001, S. 320; Jirijahn 2007, S. 307; Sedlacek 2004, S. 184). Andere Studien zeigen, dass erst eine Berufstätigkeit mit einer signifikanten Stundenanzahl die Studienzeit verlängert. Laut Body et al. (2014, S. 9) verlängert sich die Studienzeit ab acht Stunden Berufstätigkeit, wobei sich der Effekt ab 16 Stunden noch verstärkt. Zum Einfluss der Elternschaft existieren unterschiedliche Forschungsergebnisse. In Österreich und Deutschland benötigten Studierende mit Kind für das Studium länger (Helfferich et al. 2007, S. 7; Sedlacek 2004, S. 184), wohingegen beispielsweise in Frankreich offenbar kein signifikanter Effekt existiert (Body et al. 2014, S. 7). Möglicherweise hängt dies mit Länderdifferenzen zusammen, zum Beispiel in der Kinderbetreuung. Eine andere Erklärung kann die Berechnung der Studienzeit sein, da Studierende mit Kind häufig ihr Studium im Rahmen von Beurlaubungen unterbrechen und in der Beurlaubungszeit die Fachsemester nicht weiterlaufen. Somit verlängert sich die Zeit von der Einschreibung bis zum Studienabschluss, aber nicht automatisch die benötigte Studienzeit in Fachsemestern. Weitere wichtige individuelle Einflussfaktoren sind die Noten aus dem Abitur oder den ersten Studiensemestern (Aina et al. 2001, S. 320; Sedlacek 2004, S. 204; van den Berg und Hofman 2005, S. 430), Motivation (Aina et al. 2001, S. 320) und Zeitmanagement, Selbstdisziplin sowie der Austausch mit anderen Studierenden (Kuhlee et al. 2009, 45 ff.). Der Austausch mit Kommilitonen ist auch deshalb so relevant, weil die Weitergabe wichtiger Informationen häufig durch andere Studierende geschieht (Kuhlee et al. 2009, S. 53).

Institutionelle Faktoren sind studienstrukturelle oder lehrbezogene Faktoren. So zeigen van den Berg und Hofman (2005, S. 433), dass ein höherer Anteil kleinteiliger Lehrveranstaltungen die Studiengeschwindigkeit reduzieren. Wegen einer hohen Anzahl von SWS oder paralleler Fächer können Studierende sich weniger stark auf einzelne Lehrinhalte fokussieren und haben wenig Raum für Selbstlernzeit. Indirekt liefert dieses Ergebnis Hinweise darauf, dass die Selbstlernzeit mit der Studiengeschwindigkeit zusammenhängen könnte. Auch die 
Lehre an sich kann nach van den Berg und Hofman die Studiengeschwindigkeit erhöhen. So fördert aktivierende Lehre einen schnelleren Studienabschluss; allerdings ist dies nur für Studierende mit erstem Hochschulabschluss belegt. Schließlich differiert die Studiendauer je nach Studienfach (Body et al. 2014, S. 7).

Rechtliche und wirtschaftliche Faktoren außerhalb des Bildungssystems sind unter anderem Aspekte der Studienfinanzierung oder Hochschulfinanzierung. So können Veränderungen in der Studienfinanzierung den zügigen Studienabschluss forcieren. In den Niederlanden verringerten sich die Studienzeiten mit der Reduktion der staatlichen Finanzierung um ein Jahr (van den Berg und Hofman 2005, S. 415). Hohe Arbeitslosigkeit sowie eine starke Regulierung des Arbeitsmarktes führen ebenfalls zu längeren Studienzeiten, da einige Studierende ihren Abschluss aufgrund schlechter Berufsaussichten aufschieben (Aina et al. 2001, S. 321; Brunello und Winter-Ebmer 2003, S. 251).

Schließlich stellt sich die Frage, welche Studiendauer generell anzustreben ist. Die Regelstudienzeit definiert eine Anforderung an die Hochschulen, die dafür Sorge tragen müssen, dass in dieser Zeit das Studium erfolgreich absgeschlossen werden kann. Maßstab ist dabei der Durchschnittsstudent. Im Einzelfall kann es Gründe für eine längere oder kürzere Studiendauer geben, zumal beispielsweise die längere Studiendauer von erwerbstätigen Studierenden nicht mit schlechteren Noten zusammenhängt (Sprietsma 2015, S. 12).

Die oben dargestellten Studien weisen einen direkten Zusammenhang zwischen außerhochschulischen Verpflichtungen und Studienstruktur mit der Studiendauer aus. Das Modell struktureller Studierbarkeit nimmt hingegen an, dass die Studiendauer über das Studierverhalten moderiert wird, die Studierenden also ihren Besuch von Lehrveranstaltungen oder das Ablegen von Prüfungen reduzieren und sich hierdurch die Studiendauer verlängert. Diese Arbeit prüft daher den Zusammenhang zwischen subjektiven Schwierigkeiten im Studierverhalten und der geplanten Studiendauer.

H5: Schwierigkeiten bei dem Besuch von Lehrveranstaltungen und Prüfungen sowie dem Aufbringen von Selbstlernzeit hängen mit einer längeren Studiendauer zusammen.

\subsubsection{Studienzufriedenheit}

Die Studienzufriedenheit bezieht sich auf unterschiedliche Aspekte, etwa Lehrveranstaltungen, Inhalte des Studiums, Studienbelastungen und Studienbedingungen (Westermann et al. 1996, S. 12). Dabei beinhalten die Studienbedingungen auch Aspekte der Studienstruktur. Weitere Autoren 
spezifizieren den Einfluss unterschiedlicher struktureller Aspekte auf die Zufriedenheit (z. B. Blüthmann 2012, 276 ff.). Da die Studienzufriedenheit in dieser Arbeit einen Teil der theoretischen Grundlagen darstellt, sei für die detaillierteren Darstellungen auf Kapitel 2 verwiesen.

Im Sinne der Person-Environment-Fit-Theorie (Edwards et al. 1998, S. 32) können Schwierigkeiten im Studierverhalten auf eine fehlende Abstimmung zwischen den Bedürfnissen der vielfältigen Studierendenschaft und den Studienstrukturen hinweisen. Wenn die Hochschule beispielsweise wenig Blended-Learning oder Wahlmöglichkeiten anbietet und die Studierenden aufgrund ihrer Elternschaft ein hohes $\mathrm{Ma} ß$ an zeitlicher Flexibilität benötigen, kann dies die Zufriedenheit mit den Studienbedingungen und den Studienbelastungen reduzieren. Aus diesem Grund wird in dieser Arbeit der Zusammenhang zwischen den Schwierigkeiten im Studierverhalten und der Studienzufriedenheit geprüft.

H6: Schwierigkeiten bei dem Besuch von Lehrveranstaltungen und Prüfungen sowie dem Aufbringen von Selbstlernzeit hängen negativ mit der Studienzufriedenheit zusammen.

\subsection{Zusammenfassung der Hypothesen}

Die Hypothesen dieser Arbeit fokussieren das Modell struktureller Studierbarkeit. Sie analysieren, ob Studierende mit zeitlichen Restriktionen ihre Schwierigkeiten im Studierverhalten subjektiv höher einschätzen, als Studierende ohne solche Restriktionen (H2). Dabei ist interessant, ob die Studienstrukturen empirisch überprüfbar das Studierverhalten beeinflussen (H3) und welche Strukturen dies sind (H1.1). Wenn dieser Zusammenhang nachgewiesen werden kann, stellen Studienstrukturen ein geeignetes Element zur Verbesserung der Vereinbarkeit und Studierbarkeit dar. Die Hypothesen H4 bis H6 haben das Ziel, sinnvolle Ergebnisindikatoren für strukturelle Studierbarkeit zu entwickeln. Sie prüfen, welche der häufig im Qualitätsmanagement genutzten Studienerfolgsfaktoren (Studienabbruch, Studiendauer, Zufriedenheit) mit der Studierbarkeit zusammenhängen. Sämtliche oben entwickelten Hypothesen werden im Folgenden noch einmal aufgeführt und in Abbildung 9 grafisch dargestellt.

H1.1: Diejenigen Studienstrukturen, welche mit dem Studierverhalten positiv zusammenhängen, sind eine geringe Anzahl an SWS, hohe Wahlmöglichkeiten, eine gleichmäßige Verteilung von Prüfungen, freie Zeiträume für das Selbststudium und Blended-Learning. 
H2: Zeitliche Restriktionen hängen negativ mit der Teilnahme an Lehrveranstaltungen, dem Aufbringen von Selbstlernzeit und der Prüfungsteilnahme zusammen.

H3: Studienstrukturen mit hoher Flexibilität reduzieren den Zusammenhang zwischen zeitlichen Restriktionen und Problemen beim Besuch von Lehrveranstaltungen und Prüfungen sowie dem Aufbringen von Selbstlernzeit.

H4: Je mehr Schwierigkeiten Studierende bei dem Besuch von Lehrveranstaltungen und Prüfungen sowie dem Aufbringen von Selbstlernzeit haben, umso höher ist ihre Studienabbruchneigung.

H5: Schwierigkeiten bei dem Besuch von Lehrveranstaltungen und Prüfungen sowie dem Aufbringen von Selbstlernzeit hängen mit einer längeren Studiendauer zusammen.

H6: Schwierigkeiten bei dem Besuch von Lehrveranstaltungen und Prüfungen sowie dem Aufbringen von Selbstlernzeit hängen negativ mit der Studienzufriedenheit zusammen.

Die Hypothese H1.2 fokussiert die Präferenzen von Studierenden mit zeitlichen Restriktionen und prüft, ob Studierende mit mittleren und hohen zeitlichen Restriktionen flexible Studienstrukturen unflexiblen Strukturen vorziehen.

H1.2: Studierende mit zeitlichen Restriktionen präferieren Studienstrukturen und Studienformate, die ihnen zeitliche und örtliche Flexibilität ermöglichen. 


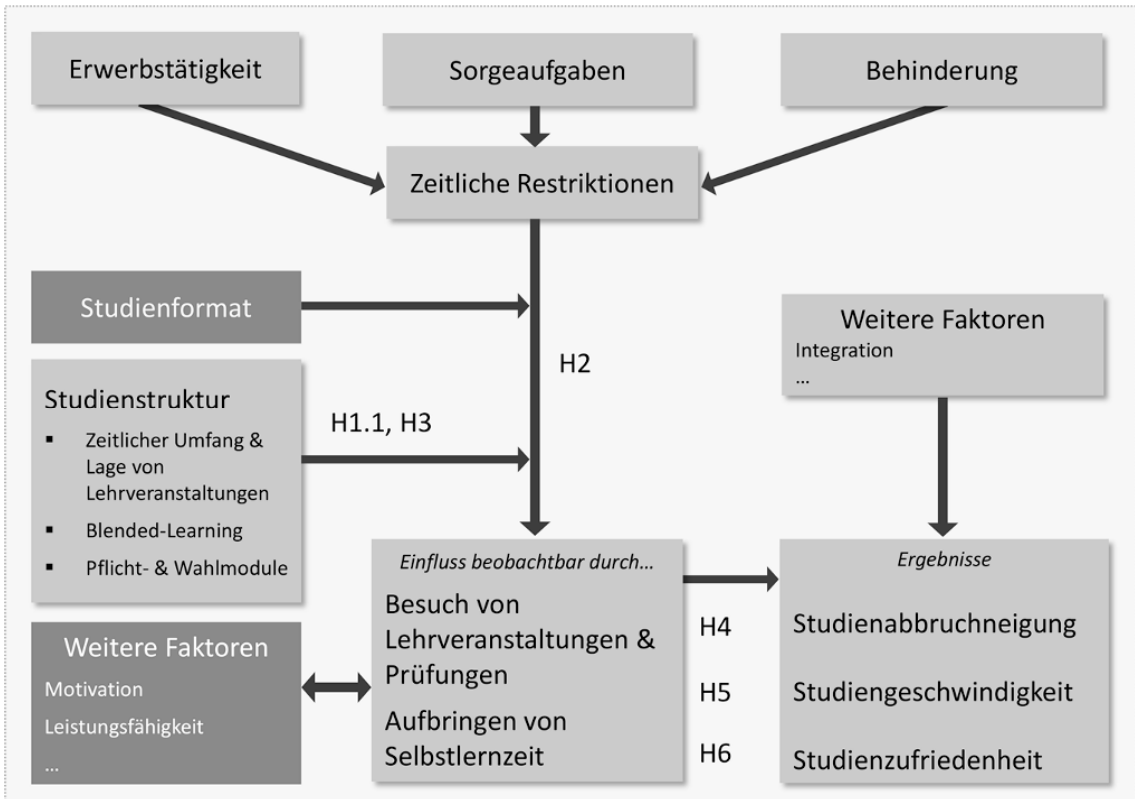

Abbildung 9 Zusammenfassung der Hypothesen zum Modell struktureller Studierbarkeit.

Open Access Dieses Kapitel wird unter der Creative Commons Namensnennung 4.0 International Lizenz (http://creativecommons.org/licenses/by/4.0/deed.de) veröffentlicht, welche die Nutzung, Vervielfältigung, Bearbeitung, Verbreitung und Wiedergabe in jeglichem Medium und Format erlaubt, sofern Sie den/die ursprünglichen Autor(en) und die Quelle ordnungsgemäß nennen, einen Link zur Creative Commons Lizenz beifügen und angeben, ob Änderungen vorgenommen wurden.

Die in diesem Kapitel enthaltenen Bilder und sonstiges Drittmaterial unterliegen ebenfalls der genannten Creative Commons Lizenz, sofern sich aus der Abbildungslegende nichts anderes ergibt. Sofern das betreffende Material nicht unter der genannten Creative Commons Lizenz steht und die betreffende Handlung nicht nach gesetzlichen Vorschriften erlaubt ist, ist für die oben aufgeführten Weiterverwendungen des Materials die Einwilligung des jeweiligen Rechteinhabers einzuholen.

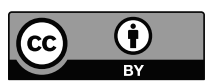

